Case Report

\title{
Acute Nontraumatic Clavicle Fracture Associated with Long-Term Bisphosphonate Therapy
}

\author{
Shen Hwa Vun, ${ }^{1}$ Yahya Husami, ${ }^{2}$ Sajan Shareef, ${ }^{1}$ and Diane Bramley ${ }^{1}$ \\ ${ }^{1}$ Trauma and Orthopaedics Department, Monklands Hospital, Monkscourt Avenue, Airdrie, North Lanarkshire ML6 0JS, UK \\ ${ }^{2}$ Department of Radiology, Monklands Hospital, Monkscourt Avenue, Airdrie, North Lanarkshire ML6 0JS, UK \\ Correspondence should be addressed to Shen Hwa Vun; shenhwavun@yahoo.co.uk
}

Received 11 June 2014; Accepted 27 July 2014; Published 31 August 2014

Academic Editor: Christian W. Muller

Copyright (c) 2014 Shen Hwa Vun et al. This is an open access article distributed under the Creative Commons Attribution License, which permits unrestricted use, distribution, and reproduction in any medium, provided the original work is properly cited.

Cases of osteonecrosis of the jaw, insufficiency fractures and atypical low energy or atraumatic fractures of pelvis, femur (subtrochanteric/mid-shaft/distal-third), tibia, fibula, metatarsal, humerus, and ulna related to long-term bisphosphonate therapy have been reported in the literature. We present the case of an acute nontraumatic clavicle fracture, associated with long-term bisphosphonate therapy, which to our knowledge has not been reported previously. This case highlights the need of critical evaluation of patients with atypical fractures during long-term bisphosphonate therapy.

\section{Introduction}

Bisphosphonates have been widely used in both the treatment and prevention of osteoporosis and osteopenia. It is well known for its efficacy in decreasing bone resorption and hypercalcemia, reducing osteolysis of bone metastasis, and its safety profile [1]. Several randomized clinical trials have also shown that bisphosphonates increase bone density and reduce the risk of vertebral, nonvertebral, and hip fractures $[2,3]$.

However, it has been suggested recently that the prolonged suppression of bone turnover under long-term administration may impair the ability of bone to remodel, leading to the accumulation of microdamage, compromised bone strength $[4,5]$, and ultimately progression to insufficiency fracture. Cases of osteonecrosis of the jaw; insufficiency fractures and low energy or atraumatic fractures of the pelvis $[6,7]$; femur involving the subtrochanteric, midshaft, and distal-third level [4, 8-13]; tibial diaphysis [14-16]; fibula [8]; metatarsal [8]; humerus [13]; and ulna [17] have been reported.

We report a case of an acute nontraumatic, transverse mid-clavicular fracture in a patient who has been on longterm alendronic acid.

\section{Case Report}

A 90-year-old postmenopausal woman presented to the accident and emergency (A\&E) department with acute pain of her right shoulder after turning the tap off in her kitchen. She reported hearing a "crack" whilst turning the tap off and noticed an obvious bump over her right shoulder, which prompted her to attend the A\&E department. Patient denied any history of previous trauma to her right clavicle, and there was no prodromal symptom such as pain over the right shoulder or clavicle.

Radiographs revealed an acute transverse right midclavicular fracture, with evidence of superior cortical thickening and a small superior spike (Figure 1). Her only past medical history was osteoarthritis of her knees and bilateral varicose veins. She had been taking treatment dose of alendronic acid $70 \mathrm{mg}$ once weekly for postmenopausal osteoporosis over the past seven years. Her other regular medication was Adcal-D3 tablets ( $1500 \mathrm{mg}$ calcium carbonate and 400 iu colecalciferol) taken once daily and paracetamol $1 \mathrm{~g}$ tablets, taken four times a day. Her most recent serum 25 hydroxyvitamin D concentration was $140 \mathrm{nmol} / \mathrm{L}$.

The patient was referred to our departmental fracture clinic a few days following her attendance in A\&E. Apart from pain on movements of her right shoulder, patient's 


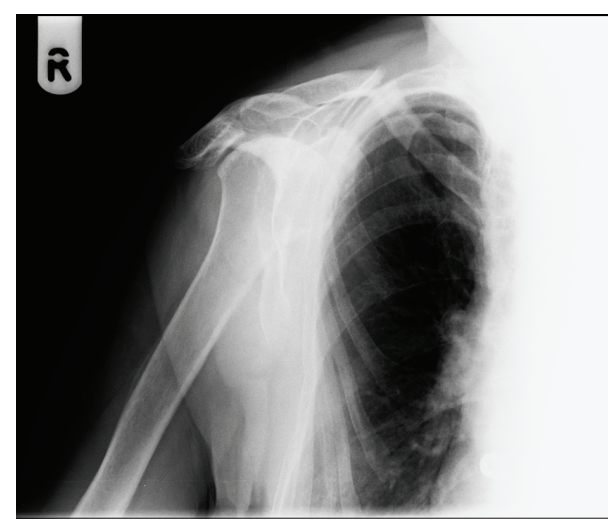

FIGURE 1: Radiograph taken in A\&E department, demonstrating acute transverse right mid-clavicular fracture.

right upper limb was neurovascularly intact. There was no evidence of skin tenting or compromise on examination. She was therefore conservatively managed on a broad arm sling. Apart from the above-mentioned past medical history, she denied any signs and symptoms of systemic illnesses or malignancy. Blood tests including myeloma screen were all normal. Her initial radiographs (Figure 1) were suggestive of an atypical fracture associated with long-term bisphosphonate treatment; therefore, both patient and her general practitioner were advised to stop the regular alendronic acid prescription.

Follow-up radiographs 4 weeks later (Figure 2) demonstrated some signs of callus formation. Figure 2 clearly demonstrates the transverse fracture and cortical thickening of the superior cortex and a small spike. At four weeks of followup, clinically, there was no gross deformity over her right shoulder. The patient's pain had reduced and she was beginning to improve her range of movement. Following discussion with the patient, a decision was made to continue treating this fracture conservatively as it would not impact her normal activities.

\section{Discussion}

Bisphosphonates are potent inhibitors of bone resorption, often prescribed as a first-line therapy for postmenopausal osteoporosis. In comparison to all other pharmacological inhibitors of bone resorption, bisphosphonates are unique for their long-term retention in the bone and the persistence of their effect after therapy cessation. Bisphosphonates have a half-life of more than 10 years [18]. However, the prolonged suppression of bone turnover may impair the ability of bone to remodel, leading to accumulation of microdamage and compromised bone strength $[4,5]$.

Atypical fractures of the femur have been described in patients receiving alendronate for more than 5 years $[10,19]$. Our patient had been taking alendronate therapy for 7 years. The incidence of these fractures was estimated to be approximately 78/100,000 in patients taking oral bisphosphonates [20]. As described by Giusti et al. [5], Odvina et al. [8], and

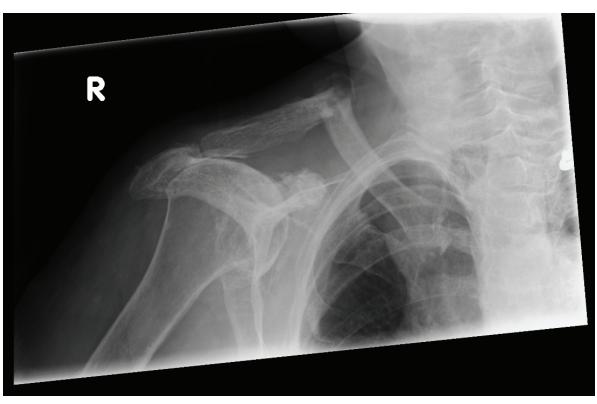

FIGURE 2: Follow-up radiograph at fracture clinic, four weeks following conservative management, demonstrating signs of callus formation of the right mid-clavicular transverse fracture. Superior cortical thickening and a small spike of the fracture fragments are also seen in this figure.

Rizzoli et al. [21], these atypical fractures occur with minimal or no trauma.

Not only does this patient have no previous history of trauma, but also the fracture occurred at an atypical site (clavicle) during the simple mechanical action of turning off a tap. We have also excluded the possibility of malignancy and systemic illnesses. Furthermore, radiographs displayed appearances similar to those reported in the literature on atypical femoral fractures associated with long-term bisphosphonates treatment: simple transverse fracture in an area of thickened cortex (superior cortex of the clavicle in this case) with unicortical beaking [8-12]. The radiograph also demonstrated degeneration of acromioclavicular joint, osteoarthritis of the right glenohumeral joint, and secondary evidence of rotator cuff arthropathy (superior subluxation of humerus); all of these could have increased the stress on the clavicle, contributing to the fracture.

Although bisphosphonates were commonly identified as important risk factors for developing atypical fractures, it is worth noting that other factors such as medications (corticosteroids and proton-pump inhibitors), cortical geometry, and properties of bone matrix have been suggested as predisposing risk factors too [22-24]. Our case report supports the observations made by others, emphasizing the need for increased awareness of atypical/stress fractures during longterm bisphosphonate therapy.

\section{Conflict of Interests}

The authors declare that there is no conflict of interests regarding the publication of this paper.

\section{References}

[1] J. Y. Reginster and N. Burlet, "Osteoporosis: a still increasing prevalence," Bone, vol. 38, supplement 1, no. 2, pp. S4-S9, 2006.

[2] S. Silverman and C. Christiansen, "Individualizing osteoporosis therapy," Osteoporosis International, vol. 23, no. 3, pp. 797-809, 2012.

[3] K. Osugi, S. Miwa, S. Marukawa, K. Marukawa, Y. Kawaguchi, and S. Nakato, "Diaphyseal femoral fatigue fracture associated 
with bisphosphonate therapy-3 more cases," Acta Orthopaedica, vol. 82, no. 1, pp. 112-113, 2011.

[4] N. B. Watts and D. L. Diab, "Long-term use of bisphosphonates in osteoporosis," Journal of Clinical Endocrinology and Metabolism, vol. 95, no. 4, pp. 1555-1565, 2010.

[5] A. Giusti, N. A. T. Hamdy, and S. E. Papapoulos, "Atypical fractures of the femur and bisphosphonate therapy. A systematic review of case/case series studies," Bone, vol. 47, no. 2, pp. 169180, 2010.

[6] K. Imai, S. Yamamoto, Y. Anamizu, and T. Horiuchi, "Pelvic insufficiency fracture associated with severe suppression of bone turnover by alendronate therapy," Journal of Bone and Mineral Metabolism, vol. 25, no. 5, pp. 333-336, 2007.

[7] V. Patel, L. Graves, and B. Lukert, "Pelvic fractures associated with long-term bisphosphonate therapy: case report," Journal of Musculoskeletal Neuronal Interactions, vol. 13, no. 2, pp. 251-254, 2013.

[8] C. V. Odvina, J. E. Zerwekh, D. S. Rao, N. Maalouf, F. A. Gottschalk, and C. Y. C. Pak, "Severely suppressed bone turnover: a potential complication of alendronate therapy," Journal of Clinical Endocrinology and Metabolism, vol. 90, no. 3, pp. 1294-1301, 2005.

[9] S. Goh, K. Y. Yang, J. S. B. Koh et al., "Subtrochanteric insufficiency fractures in patients on alendronate therapy: a caution," Journal of Bone and Joint Surgery B, vol. 89, no. 3, pp. 349-353, 2007.

[10] A. S. Neviaser, J. M. Lane, B. A. Lenart, F. Edobor-Osula, and D. G. Lorich, "Low-energy femoral shaft fractures associated with alendronate use," Journal of Orthopaedic Trauma, vol. 22, no. 5, pp. 346-350, 2008.

[11] E. B. K. Kwek, S. K. Goh, J. S. B. Koh, M. A. Png, and T. S. Howe, "An emerging pattern of subtrochanteric stress fractures: a longterm complication of alendronate therapy?" Injury, vol. 39, no. 2, pp. 224-231, 2008.

[12] Y. R. Jo, H. W. Kim, S. H. Moon, and Y. J. Ko, "A case report of long-term bisphosphonate therapy and atypical stress fracture of bilateral femur," Annals of Rehabilitation Medicine, vol. 37, no. 3, pp. 430-432, 2013.

[13] C. V. Odvina, S. Levy, S. Rao, J. E. Zerwekh, and D. S. Rao, "Unusual mid-shaft fractures during long-term bisphosphonate therapy," Clinical Endocrinology, vol. 72, no. 2, pp. 161-168, 2010.

[14] M. D. Breglia and J. D. Carter, "Atypical insufficiency fracture of the tibia associated with long-term bisphosphonate therapy," Journal of Clinical Rheumatology, vol. 16, no. 2, pp. 76-78, 2010.

[15] L. Bissonnette, P. April, R. Dumais, G. Boire, and S. Roux, "Atypical fracture of the tibial diaphysis associated with bisphosphonate therapy: a case report," Bone, vol. 56, no. 2, pp. 406409, 2013.

[16] A. M. Imbuldeniya, N. Jiwa, and J. P. Murphy, "Bilateral atypical insufficiency fractures of the proximal tibia and a unilateral distal femoral fracture associated with long-term intravenous bisphosphonate therapy: a case report," Journal of Medical Case Reports, vol. 6, article 50, 2012.

[17] K. Bjørgul and A. Reigstad, "Atypical fracture of the ulna associated with alendronate use," Acta Orthopaedica, vol. 82, no. 6, pp. 761-763, 2011.

[18] D. M. Black, A. V. Schwartz, K. E. Ensrud et al., "Effects of continuing or stopping alendronate after 5 years of treatment: the Fracture Intervention Trial long-term extension (FLEX): a randomized trial," Journal of the American Medical Association, vol. 296, no. 24, pp. 2927-2938, 2006.
[19] S. D. Vasikaran, "Association of low-energy femoral fractures with prolonged bisphosphonate use: a case-Control study," Osteoporosis International, vol. 20, no. 8, pp. 1457-1458, 2009.

[20] R. Dell, D. Greene, S. Ott et al., "A retrospective analysis of all atypical femur fractures seen in a large California HMO from the years 2007 to 2009," Journal of Bone and Mineral Research, vol. 25, supplement 1, article 1201, 2011.

[21] R. Rizzoli, K. Åkesson, M. Bouxsein et al., "Subtrochanteric fractures after long-term treatment with bisphosphonates: a European Society on Clinical and Economic Aspects of Osteoporosis and Osteoarthritis, and International Osteoporosis Foundation Working Group Report," Osteoporosis International, vol. 22, no. 2, pp. 373-390, 2011.

[22] J. Compston, "Pathophysiology of atypical femoral fractures and osteonecrosis of the jaw," Osteoporosis International, vol. 22, no. 12, pp. 2951-2961, 2011.

[23] A. Unnanuntana, A. Saleh, K. A. Mensah, J. P. Kleimeyer, and J. M. Lane, "Atypical femoral fractures: what do we know about them?: AAOS exhibit selection," Journal of Bone and Joint Surgery, vol. 95, no. 2, article e8, 13 pages, 2013.

[24] M. C. H. van der Meulen and A. L. Boskey, "Atypical subtrochanteric femoral shaft fractures: role for mechanics and bone quality," Arthritis Research and Therapy, vol. 14, no. 4, article 220, 2012. 


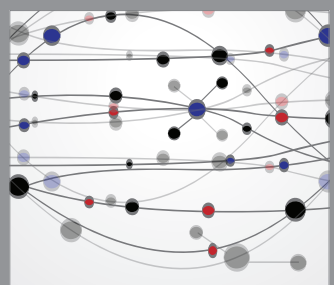

The Scientific World Journal
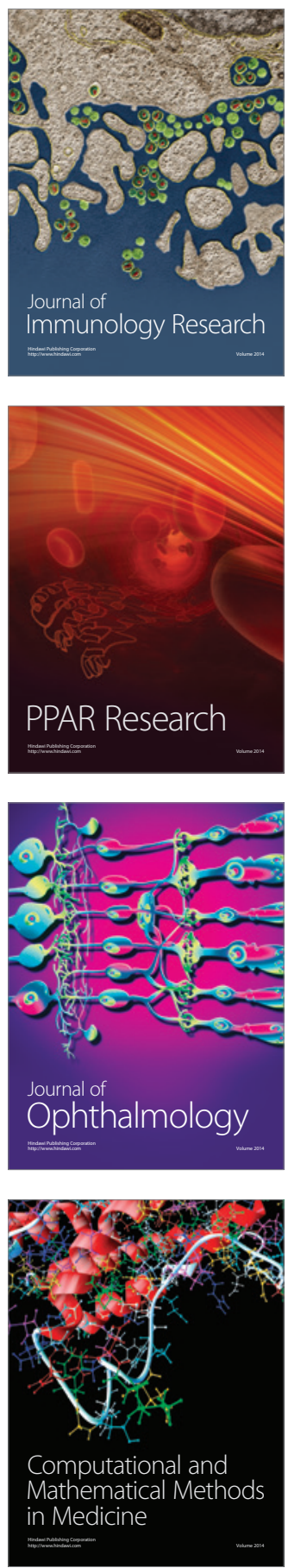

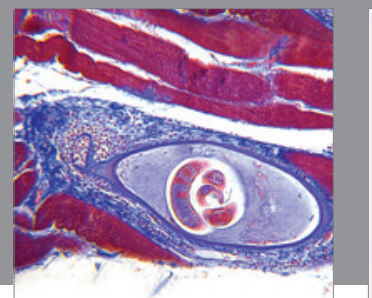

Gastroenterology

Research and Practice
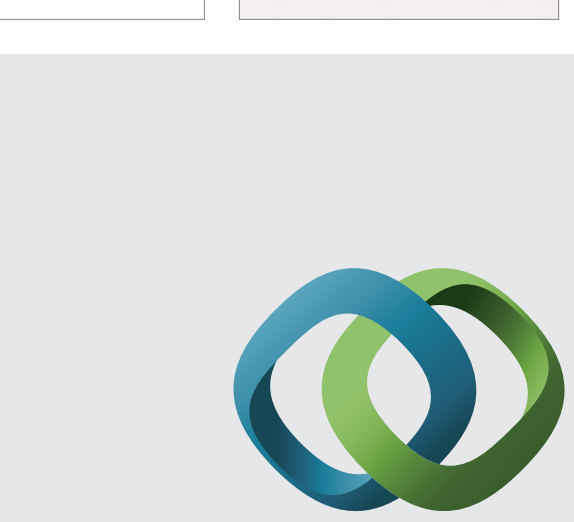

\section{Hindawi}

Submit your manuscripts at

http://www.hindawi.com
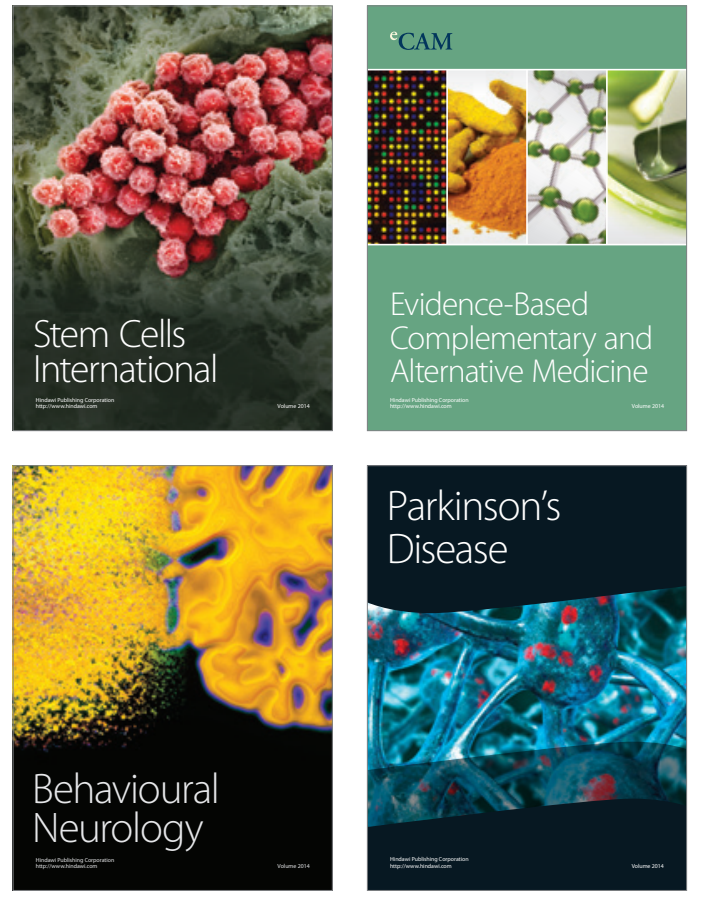
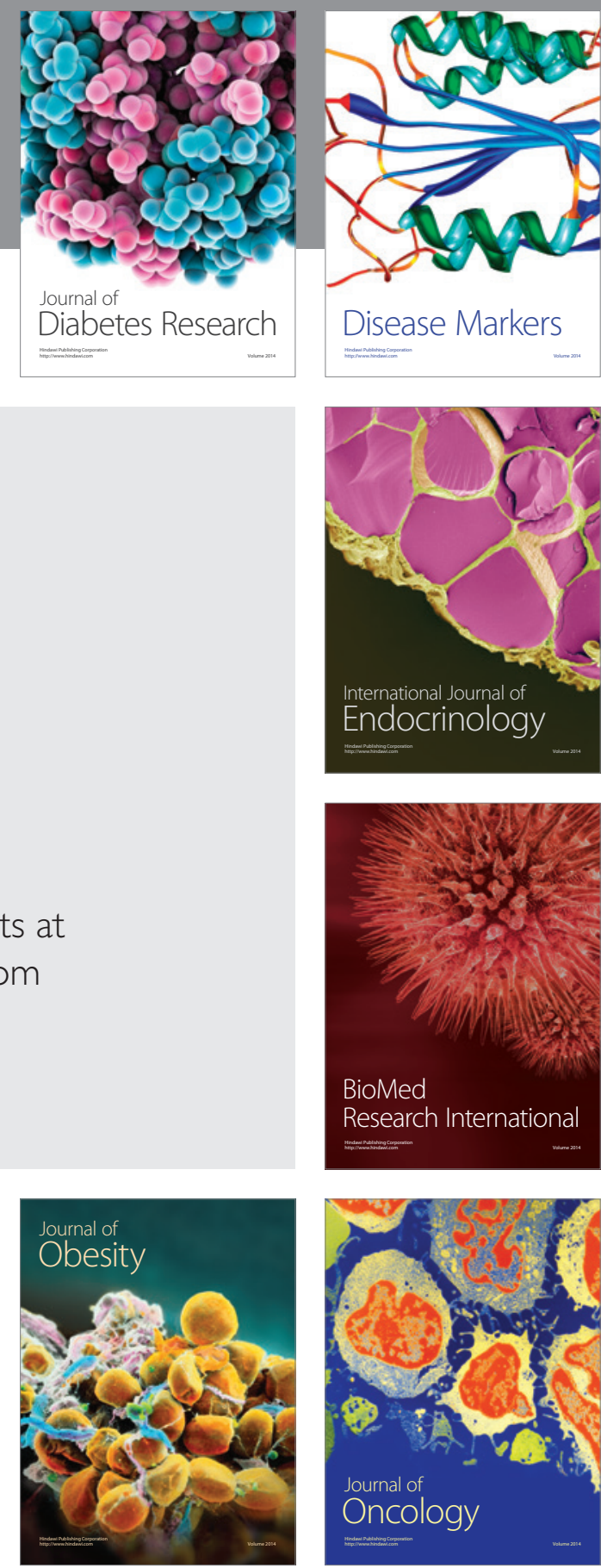

Disease Markers
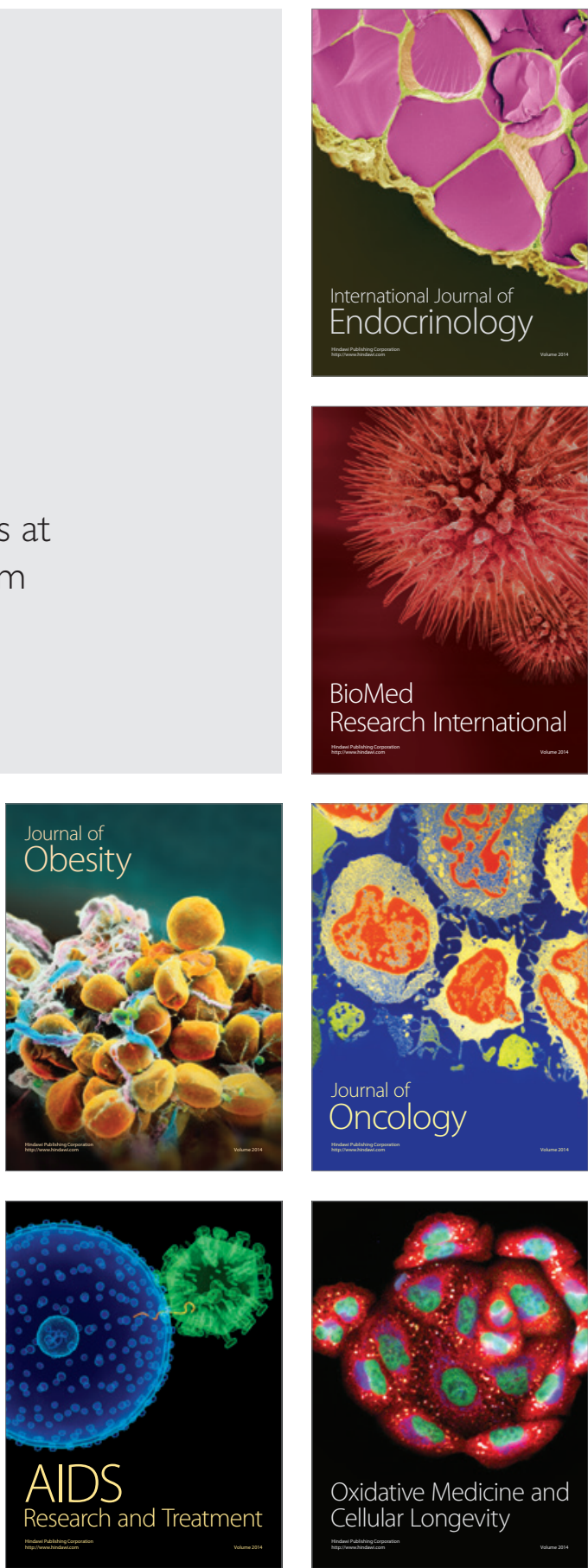